negativ. Ob dies einer von einem fraglichen Zusatznutzen begleiteten gesteigerten Sensitivität oder einer Falschpositivität anzulasten ist, muss offen bleiben.

2. Rücknahme der Radikalität in der Mammachirurgie: Dieser Trend hat auch vor der Axilladissektion nicht halt gemacht. Giuliano et al. konnten zeigen, dass bei klinischem N0-Stadium und positiven SN eine Axilladissektion keinen zusätzlichen Nutzen hat [JAMA. 2011; 305(6):569-75]. Die aktuellen Therapieempfehlungen der AGO Mamma wurden an diese Daten angepasst und eine intraoperative SN-Untersuchung nur noch dann empfohlen, wenn sie tatsächlich Konsequenzen haben sollte, was aber beim Großteil der klinisch NO-, aber pN1 (SN)-Patientinnen nicht mehr zu erwarten ist. In der Studie von Le FrèreBelda fiel mit 93\% der weit überwiegende Teil der Kohorte in diese Gruppe ohne potenzielle intraoperative Konsequenz.

„Es ist nicht unwahrscheinlich, dass von der heutigen Warte aus die Studie gar nicht mehr zustande gekommen wäre."

3. Prognostische Information statt therapeutischer Maßnahme: Wenn der therapeutische Nutzen einer Axilladissektion auch zusehends infrage gestellt wird, so bleibt doch der prognostische bestehen. Hier erscheint es sinnvoll, zwischen Makrometastasen, Mikrometastasen und Einzelzelldissemination zu differenzieren. Mit dem in der Studie von Le Frère-Belda eingesetzten Verfahren gelingt das nur sehr eingeschränkt oder gar nicht. Einzelzelldissemination erschien in 27 Fällen als negativer und in einem Fall als schwach positiver Befund. Bei vorangegangener neoadjuvanter Therapie könnte aber auch ein geringer Tumorzellnachweis noch prognostische Relevanz besitzen. Bei Komplettanalyse von SN durch OSNA droht also prognostische Information verloren zu gehen, es sei denn, es wird parallel histologisch untersucht.

4. Geschwindigkeit und Präzision: In der Mammapathologie stehen Geschwindigkeit und Präzision in reziprokem Verhältnis, und das eine wird zulasten des anderen erkauft. Daher ist die Genauigkeit eines intraoperativen Schnellschnitts der der Paraffinhistologie unterlegen. Dem Verlust an Präzision sollte ein signifikanter Nutzen gegenüberstehen. Weiterhin wird es Situ- ationen in der Mammachirurgie geben (z.B. klinisch fraglich N1), in denen zugunsten der Schnelluntersuchung entschieden und die Präzisionseinbuße durch Schnellschnitt oder OSNA in Kauf genommen werden muss. Hinsichtlich der Geschwindigkeit wird in der Studie von Le Frère-Belda und Kollegen eine Bearbeitungszeit von 40 Minuten angegeben, die damit genau das
Doppelte von dem beträgt, was die aktuelle S3 Leitlinie für die Höchstdauer eines Schnellschnittes fordert.

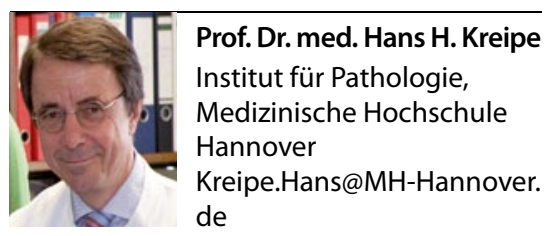

\section{Brustkrebszellen im Blut: auch ohne Metastasen ein Menetekel}

Der Nachweis von zirkulierenden Tumorzellen (CTC) im Blut von BruskrebsPatientinnen ist auch dann ein prognostisch schlechtes Zeichen, wenn noch keine Absiedlungen zu entdecken sind.

B ei etwa jeder vierten Frau mit lokalisiertem Brustkrebs ohne Lymphknotenbeteiligung kommt es nach erfolgreicher Primärtherapie zu einem systemischen Rückfall. Die dafür verantwortlichen Mikrometastasen lassen sich selbst mit hochauflösenden Bildgebungstechniken meistens nicht erkennen. Inzwischen gibt es jedoch verschiedene Tests, mit denen CTC aufgespürt werden können.

CTC sind beim metastasierten Mammakarzinom nachweislich mit einer schlechteren Prognose assoziiert. Dass sie auch für Patientinnen mit operablem Brustkrebs ohne Metastasen ein böses Omen sind, konnten Ärzte der Universität von Texas in Houston jetzt belegen.

Die Mediziner sammelten prospektiv Informationen zu CTC bei Frauen mit Brustkrebs im Stadium I-III. Bei den Frauen war jeweils nur eine Brust betroffen und in den letzten fünf Jahren kein anderes Malignom aufgetreten. Die Detektion von CTC erfolgte mit der CellSearch-Methode. Dabei werden kernhaltige Zellen aus dem peripheren Blut, die positiv für Zytokeratin und negativ für CD45 sind, durch fluoreszenzmarkierte Antikörper sichtbar gemacht.

Bei 73 von 302 Patientinnen (24\%) wurde pro 7,5 ml peripherem Blut mindestens eine CTC nachgewiesen; bei 10\% bzw. 5\% waren es sogar mindestens zwei bzw. drei CTC. Das Vorhandensein von CTC korrelierte mit keiner anderen Eigenschaft des Primärtumors, auch nicht mit dem
Lymphknotenstatus. Während der medianen Beobachtungszeit von 35 Monaten entwickelten 16 Patientinnen (5\%) Fernmetastasen und zwölf Patientinnen starben.

Bereits in dieser relativ kurzen Zeitspanne hatten Frauen mit mindestens einer CTC eine deutlich ungünstigere Prognose: Rezidive oder Todesfälle ereigneten sich bei $10 \%$ dieser Patientinnen, aber nur bei $2 \%$ der Patientinnen ohne CTC. Sowohl die Überlebenszeit ohne Wiederauftreten des Krebses als auch die Überlebenszeit überhaupt waren bei den CTC-positiven Frauen signifikant kürzer (Hazard Ratio, HR 4,62 und 4,04). Beide Ergebnisse verschlechterten sich weiter, wenn die Mindestzahl der CTC nicht eins, sondern zwei oder drei betrug.

Fazit: Der Nachweis von CTC im peripheren Blut von Brustkrebs-Patientinnen ist ein unabhängiger Prädiktor für das Risiko, einen Rückfall zu erleiden oder zu sterben. Bisher ist aber unklar, wie diese Information am besten genutzt werden kann. In den Leitlinien werden CTC weder zum Staging noch als Orientierungshilfe für Therapieentscheidungen empfohlen. Noch fehlt der Beweis, dass die Berücksichtigung von CTC auch den Behandlungserfolg verbessern kann.

Beate Schumacher

Lucci $A$ et al. Circulating tumour cells in nonmetastatic breast cancer: A prospective study. Lancet Oncol. 2012;13(7):688-95. 